\title{
Wave Equation Dispersion Inversion of Surface Waves Recorded on Irregular Topography
}

Jing Li*, Fan-Chi Lin ${ }^{\dagger}$, Amir Alam ${ }^{\dagger}$, and Gerard T. Schuster*.

* King Abdullah University of Science and Technology, Thuwal, Saudi Arabia, 23955-6900.

${ }^{\dagger}$ University of Utah, 271 Frederick Albert Sutton Building, Salt Lake City, UT 84112, USA.

\section{SUMMARY}

Significant topographic variations will strongly influence the amplitudes and phases of propagating surface waves. Such effects should be taken into account, otherwise the S-velocity model inverted from the Rayleigh dispersion curves will contain significant inaccuracies. We now show that the recently developed wave-equation dispersion inversion (WD) method naturally takes into account the effects of topography to give accurate S-velocity tomograms. Application of topographic WD to demonstrates that WD can accurately invert dispersion curves from seismic data recorded over variable topography. We also apply this method to field data recorded on the crest of mountainous terrain and find with higher resolution than the standard WD tomogram.

\section{INTRODUCTION}

There are a number of studies that demonstrate surface waves can strongly scatter from topographic variations along the recording surface (Davies and Heathershaw, 1984; Snieder, 1986; Spetzler et al., 2002; Nuber et al., 2016; Borisov et al., 2016). Unless these topographic effects are taken into account, the Svelocity model inverted from the surface waves will contain significant inaccuracies. As an example, Figure 1a depicts a velocity model with several local variations of the S-velocity and an irregular topographic surface (yellow filled line). We used a 2D elastic finite-difference algorithm (Robertsson, 1996; Wang et al., 2015) to compute the vertical-component shot gather shown in Figure $1 \mathrm{~b}$ for sources and receivers on the free surface. This compares to the traces in Figure 1c recorded along a horizontal free surface denoted by the yellow dashed line in Figure 1a. It is obvious that there are noticeable differences between the shot gather in Figure 1b compared to the one recorded over a horizontal surface in Figure 1c.

Li and Schuster (2016) developed a method for inverting dispersion curves associated with surface waves. This method is denoted as wave equation dispersion inversion (WD) and has the benefit of robust convergence compared to the tendency of full waveform inversion (FWI) to getting stuck in local minima (Masoni et al., 2014; Solano et al., 2014; Yuan et al., 2015; Köhn et al., 2016). It has the advantage over the traditional inversion of dispersion curves (Xia et al., 1999; Socco et al., 2010; Maraschini et al., 2010) in that is does not assume a layered model and is valid for arbitrary 2D or 3D media. The standard WD method also can account for topographic effects by incorporating the free-surface topography into the finitedifference solution to the elastic wave equation. We will denote the WD method as the topographic WD (TWD) if it takes topography into account by solving the elastic wave equation
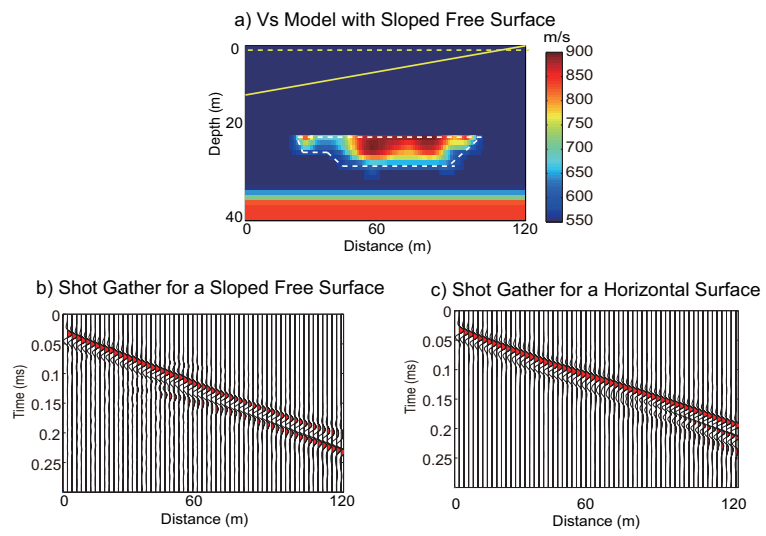

Figure 1: a) S-velocity model with strong topographic variations similar to the topography in the Southern California field experiment. The yellow line is the topographic surface and the white dashed line is the horizontal surface. b) Typical shot gather for vertical-component records computed by a 2-4 finite-difference solution to the $2 \mathrm{D}$ elastic wave-equation with a free surface having variable topography, d) shot gather computed for a horizontal free surface along the dashed line in a). c) S-velocity model inverted from the dispersion curves computed from 30 shot gathers with shots along the topographic surface in a) and 60 vertical-component receivers located every $2 \mathrm{~m}$ along the surface.

for sources and receivers on the actual topography of the free surface. In this paper we validate the TWD method for surface waves recorded on free surfaces with strong variations in topography. Firstly, we briefly review the theory of WD, with mathematical details provided in $\mathrm{Li}$ et al. (2017). This section also provides the workflow for implementing the TWD method for traces recorded on irregular topography. The next section presents numerical results that validate the TWD method for synthetic data and field data recorded along a line with significant topography in Southern California. The topography of the recording surface in both the synthetic and field data examples is similar to one another with an elevation change of over 300 $\mathrm{m}$ along a recording line with length $2.5 \mathrm{~km}$. The final section presents conclusions.

\section{THEORY}

The WD method (Li et al., 2017) inverts for the S-velocity model that minimizes the dispersion misfit function $\varepsilon$ :

$$
\varepsilon=\frac{1}{2} \sum_{\omega}(\overbrace{\kappa(\omega)-\kappa(\omega)^{o b s}}^{\text {residual }=\Delta \kappa(\omega)})^{2},
$$




\section{WD with Topography}

where, $\kappa(\omega)$ represents the predicted dispersion picked from the simulated spectrum and $\kappa(\omega)^{o b s}$ denotes the dispersion curve obtained from the recorded spectrum. In practice, these spectra are computed by applying a linear Radon transform (LRT) to the common shot gather in the frequency domain (Li and Schuster, 2016). Any order of dispersion curve can, in principle, be picked and inverted, but for the examples in this paper we only use the fundamental mode of Rayleigh waves.

The slowness gradient $\gamma(\mathbf{x})$ of equation 1 is given by

$$
\gamma(\mathbf{x})=\frac{\partial \varepsilon}{\partial s(\mathbf{x})}=\sum_{\omega} \Delta \kappa(\omega) \frac{\partial \kappa(\omega)}{\partial s(\mathbf{x})},
$$

and the optimal shear-slowness model $s(\mathbf{x})$ is obtained from the steepest-descent formula:

$$
s(\mathbf{x})^{(k+1)}=s(\mathbf{x})^{(k)}-\alpha \sum_{\omega} \Delta \kappa(\omega) \frac{\partial \kappa(\omega)}{\partial s(\mathbf{x})},
$$

where $\alpha$ is the step length by any backtracking line-search method (Nocedal and Wright, 1999) and the superscript $(k)$ denotes the $k^{\text {th }}$ iteration. For pedagogical simplicity, we assume a single shot gather $\hat{D}(\mathbf{g}, \omega)$ for a source at $\mathbf{s}$ and geophones at $\mathbf{g}$, and the notation for the source location is silent. The misfit function will include an additional summation over different shot gathers if more than one shot gather is used. Mathematical details for deriving the Fréchet derivative $\frac{\partial \kappa(\omega)}{\partial s(\mathbf{x})}$ are given in Li et al. (2017). The interpretation of equation 3 is that the traces and source wavelet are weighted by terms proportional to the wavenumber residual $\Delta \kappa(\omega)$ and backpropagated into the medium to update the slowness of the S-velocity model.

\section{Workflow for WD with Topography}

The steps for implementing the TWD algorithm are the following.

1. Mute the body waves and higher-order modes of the Rayleigh-waves in the observed and predicted shot gathers. Then apply a 1D Fourier transform along the time axis of the shot gather to get the frequency spectrum of each trace in the shot gather. The predicted shot gather is computed by a 2D finite-difference solution to the elastic wave equation (Zeng et al., 2012; Wang et al., 2012) for shots and receivers on an irregular free surface.

2. Apply a linear Radon transform (LRT) to the frequency spectra of the predicted and observed shot gathers to get the phase-velocity curve $C(\omega)$ of the fundamental Rayleigh mode. Here, the finite-difference modeling of the elastic wave equation is for sources and receivers on an irregular free surface. The fundamental dispersion curves are automatically picked according to the maximum amplitudes of the magnitude spectrum that are nearest to the slowness axis. Details for picking the dispersion curves are in Li et al. (2017).

3. Calculate the weighted data $D(\mathbf{g}, \omega)$, which are then used for computing the backprojected data. The forward propagated source wavelet is weighted by the residual $\Delta \kappa(\omega)$

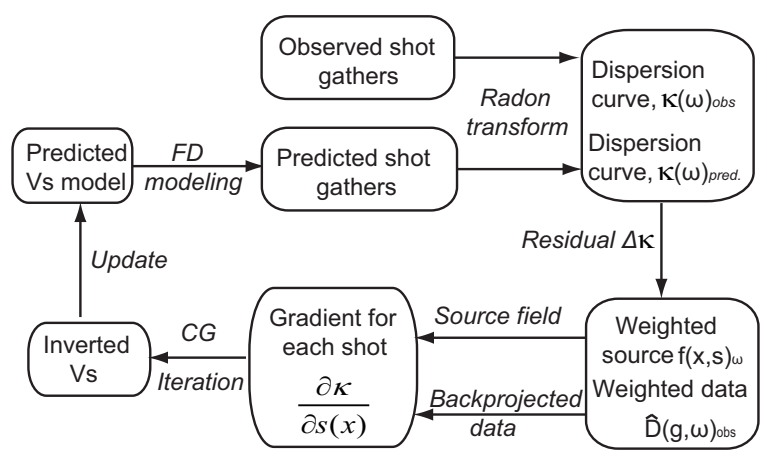

Figure 2: The workflow for implementing the WD method.

4. Estimate the step-length $\alpha$ by any backtracking linesearch method.

5. The gradients for each migrated shot gather are added together to get the S-velocity update. The background S-velocity model is updated and the above steps are repeated until the RMS residual falls below a specified value.

\section{NUMERICAL TESTS}

The effectiveness of the WD method is now demonstrated with synthetic and field data examples, where the data are recorded on surfaces with significant variations in topography. The synthetic example is a complex topographic model with both canyons and horst-like features beneath the free surface. The field data example is for ambient noise data recorded in Southern California. In the synthetic examples, the observed data are generated by a staggered-grid solution of the $2 \mathrm{D}$ elastic waveequation for a free surface with strong topographic variations (Zeng et al., 2012). In these examples, the P-wave velocity is updated by assigning $v_{p}=\sqrt{3} v_{s}$ and the density is taken to be a constant value of $1500 \mathrm{~g} / \mathrm{cm}^{3}$. The WD tomograms that neglect topography are computed from predicted data with sources and receivers on a horizontal free surface. In such cases the predicted data do not take into account the phase and velocity variations in the observed surface waves recorded on the irregular recording topography.

\section{Synthetic Data}

Two sets of synthetic data are generated for the S-velocity model in Figure 1a: set A is for shot gathers computed for sources and receivers on the horizontal free surface (dashed yellow line) and set B is for shots and receivers on the sloped free surface (solid yellow line).

If the input data are from set A and the WD method computes data for that free surface, then the resulting S-velocity tomogram in Figure $3 \mathrm{a}$ resembles that from the actual model. However, if the input data are from set B and the WD method computes the predicted data for sources and receivers on a horizontal free surface then the resulting tomogram in Figure $3 b$ contains significant errors. 


\section{WD with Topography}
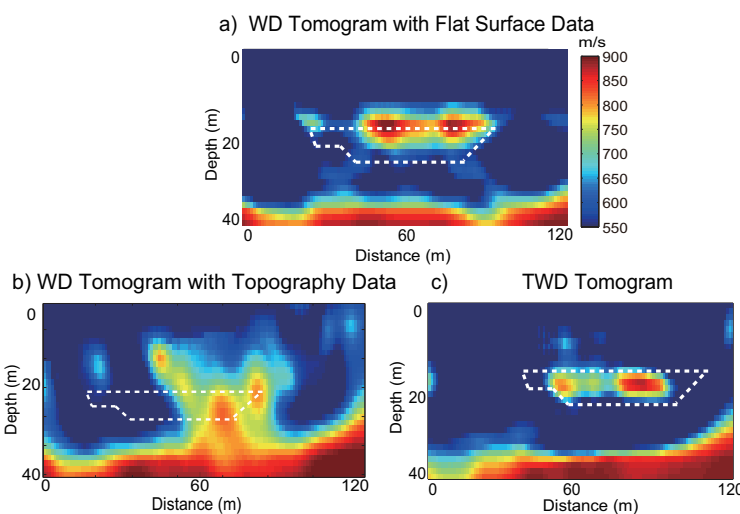

Figure 3: a) WD tomograms where the input data are recorded along a) a free surface and b) an irregular free surface. The TWD tomogram is computed from data recorded along the irregular free surface.

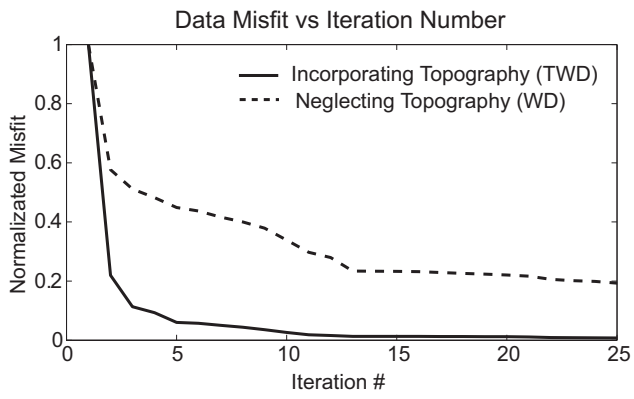

Figure 4: Plot of residual vs iteration number for the synthetic examples. The Y-axis represents the normalized frequencyshift data residual, and the solid and dashed lines represent the WD results that, respectively, take into account and neglect the effects of an irregular free surface.

To eliminate these topographic errors, the WD method can generate the predicted data for sources and receivers on the actual irregular free surface. We will denote this as the topographic WD (TWD) method. The resulting TWD tomogram is shown in Figure 3c, where the location of the blue velocity anomalies mostly agrees that in the actual model.

In addition, Figure 4 shows that after 25 iterations, the normalized TWD residual (black full line) is less than the WD residual that neglects topographic variations (black dashed line). It indicates that the inverted traces associated with the TWD tomogram more closely resemble the observed ones.

\section{Field Data Tests}

The TWD and WD methods are now tested on ambient noise data recorded along the Clark strand of the San Jacinto fault zone in Southern California, USA (Figures 5a). The location of the field experiment is shown in Figure 5a (red square) and the white dashed line denotes the main fault which is aligned along the west-north direction. Figure $5 \mathrm{~b}$ shows the location of each recording station across the fault in a month-long deployment of a linear array of 134 Fairfield three-component 5- $\mathrm{Hz}$

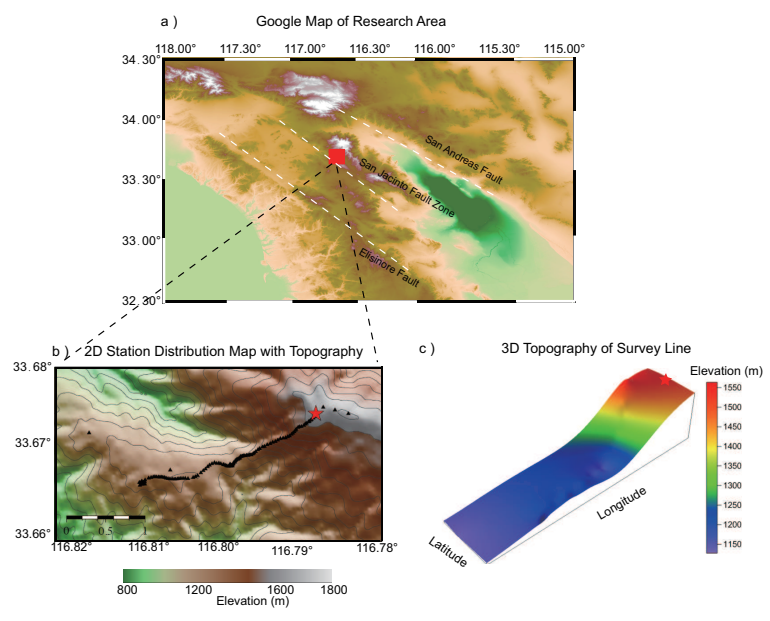

Figure 5: The survey line where the ambient noise is recorded along the crest of a mountain in Southern California. Illustration a) is the Google map of the research region, b) is the 2D station distribution map and c) depicts the 3D topographic map associated with the survey line.

seismometers. With a total aperture of $2.4 \mathrm{~km}$ and a mean station spacing of $20 \mathrm{~m}$, the array locally spans the fault zone from a low-velocity crustal block on the South-West (SW) through the damage structure of the fault to a high-velocity crustal block on the North-East (NE) (Allam and Ben-Zion, 2012; Share et al., 2017). The data were continuously recorded for 36 days at a $1000-\mathrm{Hz}$ sampling rate. Figure 5c shows the irregular topography along the survey line, where the largest elevation difference is about $300 \mathrm{~m}$. Before the TWD inversion, the following processing steps are applied to the raw ambient-noise data (Bensen et al., 2007; Lin et al., 2007)

- Remove instrument response, remove mean, remove trend, band-pass filter, and segment the entire trace to a sequence of shorter traces.

- Apply time domain normalization and spectral whitening.

- Cross-correlation and temporal stacking of the traces (Lin et al., 2008).

A virtual shot gather for a virtual shot at an endline recording station is shown in Figure 6a. Adding the time-reversed acausal portion of the trace with the causal portion gives the result shown in Figure 6b. There is strong energy near the zero time which is likely caused by body waves for local earthquakes. The shot gathers are muted so only the strongest surface waves are retained, as shown in Figure 6c. The dispersion curve associated with the shot gather with the source at station 2 is shown in Figure 6d.

The virtual shot gathers consist of 125 common shot gathers (CSGs) with sources located an average of $20 \mathrm{~m}$ along the recording line. Each shot is recorded by 125 receivers with an average spacing of $20 \mathrm{~m}$. Figure $7 \mathrm{a}$ shows the standard WD S-velocity tomogram that neglects topography, and indicates that there is a low-velocity zone for the offset range 

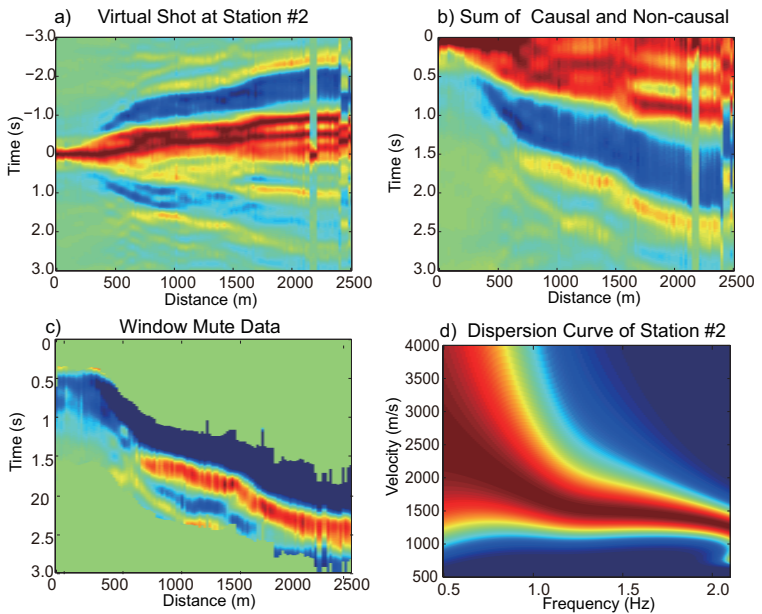

Figure 6: Data processing results for the Southern California data. a) Virtual shot gather at station No. 2, b) the sum of the causal and time-reversed non-causal parts of the shot gather, c) data after windowing, d) and the dispersion curve for station No. 2.

$900 m<x<1400 m$, which is generally consistent with the local damaged structure of the San Jacinto fault zone. For comparison, the TWD tomogram is shown in Figure $7 \mathrm{~b}$ and shows an obvious low-velocity anomaly that is coincides with that seen in the WD tomogram. In addition, the TWD tomogram shows more consistent across the fault and higher resolution features shows more clearly the velocity contrast across the fault and not seen in the the WD tomogram.

As a final check, Figure 7c shows a common offset gather (COG) with the source-receiver offset of $200 \mathrm{~m}$. The white dashed line in this figure indicates the location of the main fault at the offset range $900 m<\mathrm{x}<1000 \mathrm{~m}$. Compared to the WD tomogram, the locations of the low-velocity anomalies in the TWD tomogram are more consistent with the interpreted faults (white lines) in the COG profile. For example, there are strong indications of antithetic faulting seen in the TWD tomogram and COG not seen in the WD tomogram.

\section{DISCUSSION AND CONCLUSIONS}

We present the TWD method that accounts for an irregular recording surface by using elastic finite-difference solutions for sources and receivers on the irregular free surfaces. The effectiveness of this method is numerically demonstrated with synthetic data and field traces recorded on an irregular free surface. Results with synthetic data suggest that there will be significant errors in the tomogram if topographic phase delays larger than a quarter of a cycle are not accounted for in the inversion. As an empirical rule, we use TWD when the topographic elevation varies by more than a quarter of a wavelength for source-receiver offsets less than 3-4 wavelengths. Appropriate corrections to this rule can be determined by synthetic simulations for sources and receivers on the specified survey topography. The TWD method is also applied to field data
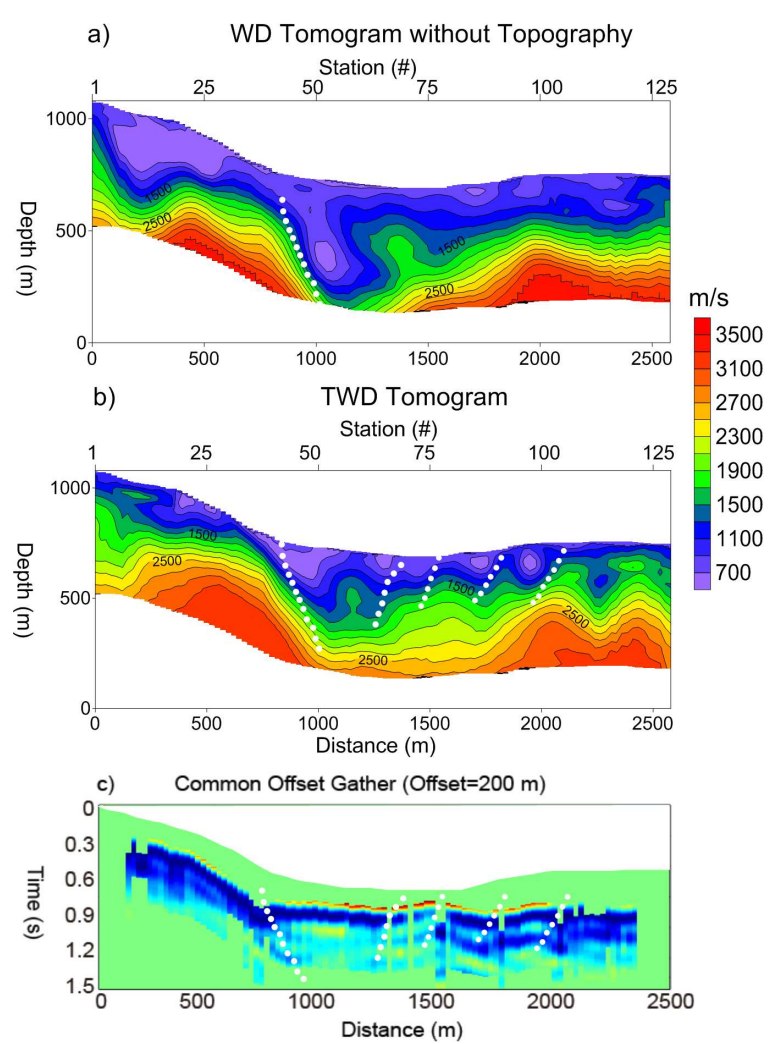

Figure 7: a) S-velocity tomogram inverted from the Southern California dispersion curves, b) S-velocity tomogram obtained by the TWD method that takes into account the irregular topography, and c) COGs at the source-receiver offset of $200 \mathrm{~m}$. The dashed white faults are the interpreted faults, where the dashed line at the far left is the main fault seen at the surface.

recorded over mountainous terrain in Southern California. The S-velocity TWD tomogram shows more fault-like details than the WD tomogram, and some fault-like structures appear to more consistent with those seen in the COG image. However, the actual sub-surface fault model is not except for the location of the main fault.

\section{ACKNOWLEDGEMENTS}

We thank the sponsors for supporting the Consortium of Subsurface Imaging and Fluid Modeling (CSIM). We also thank the KAUST provide the funding by the CRG grant OCRF2014-CRG3-2300. 
EDITED REFERENCES

Note: This reference list is a copyedited version of the reference list submitted by the author. Reference lists for the 2017 SEG Technical Program Expanded Abstracts have been copyedited so that references provided with the online metadata for each paper will achieve a high degree of linking to cited sources that appear on the Web.

\section{REFERENCES}

Allam, A., and Y. Ben-Zion, 2012, Seismic velocity structures in the Southern California plate-boundary environment from double difference tomography: Geophysical Journal International, 190, 11811196, http://doi.org/10.1111/j.1365-246X.2012.05544.X.

Bensen, G., M. Ritzwoller, M. Barmin, A. Levshin, F. Lin, M. Moschetti, N. Shapiro, and Y. Yang, 2007, Processing seismic ambient noise data to obtain reliable broad-band surface wave dispersion measurements: Geophysical Journal International, 169, 1239-1260, http://doi.org/10.1111/j.1365-246X.2007.03374.X.

Borisov, D., R. Modrak, H. Rusmanugroho, Y. Yuan, F. Simons, J. Tromp, and F. Gao, 2016, Spectralelement based 3D elastic full-waveform inversion of surface waves in the presence of complex topography using an envelope-based misfit function: 86th Annual International Meeting, SEG, Expanded Abstracts, 1211-1215, http://doi.org/10.1190/segam2016-13843759.1.

Davies, A., and A. Heathershaw, 1984, Surface-wave propagation over sinusoidally varying topography: Journal of FluidMechanics, 144, 419-443, http://doi.org/10.1017/S0022112084001671.

Kohn, D., T. Meier, M. Fehr, D. De Nil, and M. Auras, 2016, Application of 2D elastic Rayleigh waveform inversion to ultrasonic laboratory and field data: Near Surface Geophysics, 14, 461476, http://doi.org/10.3997/1873-0604.2016027.

Li, J., and G. Schuster, 2016, Skeletonized wave equation of surface wave dispersion inversion: 86th Annual International Meeting, SEG, Expanded Abstracts, 3630-3635, http://doi.org/10.1190/segam2016-13770057.1.

Li, J., Z. Feng, and G. T. Schuster, 2017, Wave-equation dispersion inversion: Geophysical Journal International, 208, 1567-1578, http://doi.org/10.1093/gji/ggw465.

Lin, F.-C., M. H. Ritzwoller, J. Townend, S. Bannister, and M. K. Savage, 2007, Ambient noise Rayleigh wave tomography of New Zealand: Geophysical Journal International, 170, 649-666, https://doi.org/10.1111/j.1365-246X.2007.03414.X.

Lin, F.-C., M. P. Moschetti, and M. H. Ritzwoller, 2008, Surface wave tomography of the Western United States from ambient seismic noise: Rayleigh and Love wave phase velocity maps: Geophysical Journal International, 173, 281-298, https://doi.org/10.1111/j.1365-246X.2008.03720.x.

Maraschini, M., F. Ernst, S. Foti, and L. V. Socco, 2010, A new misfit function for multimodal inversion of surface waves: Geophysics, 75, no. 4, G31-G43, https://doi.org/10.1190/1.3436539.

Masoni, I., R. Brossier, J. Boelle, and J. Virieux, 2014, Generic gradient expression for robust FWI of surface waves: 76th Annual International Conference and Exhibition, EAGE, Extended Abstracts, 1-5, https://doi.org/10.3997/2214-4609.20141407.

Nocedal, J., and S. Wright, 1999, Numerical optimization: Springer Series in Operations Research: Springer Company, 35.

Nuber, A., E. Manukyan, and H. Maurer, 2016, Ground topography effects on near-surface elastic full waveform inversion: Geophysical Journal International, 207, 67-71, https://doi.org/10.1093/gji/ggw267.

Robertsson, J. O., 1996, A numerical free-surface condition for elastic/viscoelastic finite-difference modeling in the presence of topography: Geophysics, 61, 1921-1934, https://doi.org/10.1190/1.1444107. 
Share, P.-E., Y. Ben-Zion, Z. E. Ross, H. Qiu, and F. Vernon, 2017, Internal structure of the San Jacinto fault zone at Blackburn Saddle from seismic data of a dense linear array: Geophysical Journal International, in review.

Snieder, R., 1986, The influence of topography on the propagation and scattering of surface waves: Physics of the Earth and Planetary Interiors, 44, 226-241, https://doi.org/10.1016/00319201(86)90072-5.

Socco, L. V., S. Foti, and D. Boiero, 2010, Surface-wave analysis for building near-surface velocity models established approaches and new perspectives: Geophysics, 75, no. 5, A83-A102, https://doi.org/10.1190/1.3479491.

Solano, C. P., D. Donno, and H. Chauris, 2014, Alternative waveform inversion for surface wave analysis in 2-Dmedia: Geophysical Journal International, 198, 1359-1372, https://doi.org/10.1093/gji/ggu211.

Spetzler, J., J. Trampert, and R. Snieder, 2002, The effect of scattering in surface wave tomography: Geophysical Journal International, 149, 755-767, https://doi.org/10.1046/j.1365246X.2002.01683.X.

Wang, L., Y. Luo, and Y. Xu, 2012, Numerical investigation of Rayleigh-wave propagation on topography surface: Journal of Applied Geophysics, 86, 88-97, https://doi.org/10.1016/j.jappgeo.2012.08.001.

Wang, L., Y. Xu, J. Xia, and Y. Luo, 2015, Effect of near-surface topography on high-frequency Rayleigh-wave propagation: Journal of Applied Geophysics, 116, 93-103, https://doi.org/10.1016/j.jappgeo.2015.02.028.

Xia, J., R. D. Miller, and C. B. Park, 1999, Estimation of near-surface shear-wave velocity by inversion of Rayleigh waves: Geophysics, 64, 691-700, https://doi.org/10.1190/1.1444578.

Yuan, Y. O., F. J. Simons, and E. Bozdag, 2015, Multiscale adjoint waveform tomography for surface and body waves: Geophysics, 80, no. 5, R281-R302, https://doi.org/10.1190/GEO20140461.1.

Zeng, C., J. Xia, R. D. Miller, and G. P. Tsoflias, 2012, An improved vacuum formulation for 2D finitedifference modeling of Rayleigh waves including surface topography and internal discontinuities: Geophysics, 77, no. 1, T1-T9, https://doi.org/10.1190/geo2011-0067.1 